Research Article

\title{
Target Tracking Algorithm of Basketball Video Based on Improved Grey Neural Network
}

\author{
You Jun Wang ${ }^{1,2}$ and Guo Huang $\mathbb{D}^{1,2}$ \\ ${ }^{1}$ Jiaozuo University, Department of Taichi and Martial Arts, Jiaozuo 454002, China \\ ${ }^{2}$ Zhejiang Wanli University, Ningbo 315100, Zhejiang, China
}

Correspondence should be addressed to Guo Huang; 2002900057@zwu.edu.cn

Received 10 August 2021; Revised 4 September 2021; Accepted 27 September 2021; Published 27 October 2021

Academic Editor: Bai Yuan Ding

Copyright (C) 2021 You Jun Wang and Guo Huang. This is an open access article distributed under the Creative Commons Attribution License, which permits unrestricted use, distribution, and reproduction in any medium, provided the original work is properly cited.

\begin{abstract}
Considering the limitation of tracking range on sports video target tracking in basketball games, there are some problems, such as poor tracking effect, low accuracy, low anti-interference ability, and being time-consuming. Therefore, this study proposes a target tracking algorithm of basketball video based on improved grey neural network. According to the pixel grey difference of the target image in basketball video, this study applies the adaptive threshold algorithm in order to segment the target image of basketball video and obtain the target area of basketball video. This algorithm can normalize the grey level of the target area, build the generating sequence of the target area, and collect the target data of the basketball video. It obtains the feature output matrix of basketball video target based on the geometric dispersion of the target image and extracts the key feature points of basketball video target by single frame visual difference analysis. In addition, it makes use of the improved grey neural network to track and locate the feature points of basketball video target and reconstruct the basketball video target image with superresolution to realize basketball video target tracking. The experimental results show that the proposed algorithm has good target tracking effect of basketball video, can effectively improve the target tracking accuracy and anti-interference ability, and can shorten the target tracking time.
\end{abstract}

\section{Introduction}

As it is known, the development of computer network technology promotes the update of image processing technology, and the video processing technology is also applied in people's daily lives [1,2]. At present, the image accuracy of basketball video is high, but due to the variable target trajectory of basketball video, the similar moving target, and complex background, it is more difficult to track the target of basketball video. In addition, in the actual basketball game, it is difficult for the target tracking effect to achieve the ideal state due to the influence of various factors such as illumination, occlusion, and the definition of image acquisition equipment [3]. Therefore, the research on target tracking algorithm of basketball video has become a hot issue.

Yang and Wang [4] proposed a target tracking algorithm of basketball video based on DSP and FPGA. This study constructed a target index of the basketball video on the basis of collecting the basketball video, initialized the video, and determined the basic color map of the target index. Then according to the input basketball image, it used FPGA technology to obtain a new target index image and processed the new target image and the original image to get a new target position, so as to realize the target tracking of basketball video. The algorithm in this study can effectively track moving targets in basketball game video, but the tracking accuracy of moving targets is low. Wang and Hou [5] proposed a target tracking algorithm of sports video based on optimized particle filter. This study firstly detects the sports video, removes the background color histogram, then tracks the color target by using the particle filter technology, combines it with the color distribution, and finally realizes the target tracking of sports video by optimizing the tracked target model. The algorithm has high efficiency, but poor performance in target tracking of 
moving video. Aiming at solving the above problems, this study proposes a target tracking algorithm of basketball video based on improved grey neural network, which provides a certain reference for accurately tracking basketball video target.

\section{Design of Target Tracking Algorithm in Basketball Video}

2.1. Obtaining the Target Area of Basketball Video. In order to obtain the target area of basketball video, this study assumes that the pixel point of basketball video image is $(x, y)$ and sets the grey neural network as $I(x, y, t)$, and the time is $t$. At time $t+1, I(x, y, t+1)$ is used to represent the grey neural network. The pixel grey difference at time $t$ and time $t+1$ is calculated by the following formula, which is expressed as

$$
\Delta I(x, y)=\{I(x, y, t+1)-I(x, y, t)\} .
$$

By iterative processing of formula (1), it can obtain the segmentation threshold $T$ of basketball video that is processed by improved grey neural network and play the advantages of visual technology [6-8] to obtain the possible areas of the moving target in the basketball video:

$$
I(x, y)= \begin{cases}1, & \Delta I(x, y)>T \\ 0, & \Delta I(x, y) \leq T\end{cases}
$$

Particle filter is used to detect the target of the processed basketball video [9-11]. In order to increase the accuracy, it is assumed that when the basketball video is at time $t$, the pixel point $(x, y)$ of the image is represented by grey neural network $I(x, y, t)$. After simulation of the motion of basketball video, if this pixel point remains unchanged, when $d I / d t=0$, it can obtain the following formula:

$$
u I_{x}+v I_{y}+I_{t}=0 .
$$

In formula (3), $u=d x / d t, v=d y / d t$, and the differentiation of the target area images $x, y$, and $t$ in the basketball video can be represented by $I_{x}, I_{y}$, and $I_{t}$.

It can obtain the solution of $(u, v)$ by using the iterative algorithm:

$$
\begin{aligned}
& u_{x, y}^{k+1}=u_{x, y}^{-k}-\frac{I x\left(I x u_{x, y}^{-k}+I y v_{x, y}^{-k}+I t\right)}{\Delta I(x, y)^{2}+I x^{2}+I y^{2}} \\
& v_{x, y}^{k+1}=v_{x, y}^{-k}-\frac{I y\left(I x u_{x, y}^{-k}+I y v_{x, y}^{-k}+I t\right)}{a^{2}+I x^{2}+I y^{2}} .
\end{aligned}
$$

Here, $a$ represents the iterative coefficient.

Referring to formulas (4) and (5), it can segment the basketball video image by using the threshold after iterative processing:

$$
\operatorname{Energyd}\left(I_{i}, C_{j}\right)=\left((u)^{2}+(v)^{2}\right)^{1 / 2} .
$$

Here, $I_{i}$ represents the area where the target may appear and $C_{j}$ represents the area where the target actually appears.
Taking clustering analysis of the segmented image by the image processing technology [12-14], it can obtain the target area of the basketball video, which can be shown in the following formula:

$$
j=\min \left\{j / d\left(I_{i}, C_{j}\right)\right\} \leq \theta_{1} .
$$

In formula (7), the distance between the two areas is $d\left(I_{i}, C_{j}\right)$, the maximum distance threshold of the two target areas of basketball video is $\theta_{1}$, and the nonzero pixel in the basketball video is $I_{i}$.

2.2. Acquisition of Target Data in Basketball Video. The algorithm in this study fuses the feature vectors by matching technology, monitors three-dimensional fusion target in the target area of basketball video, reconstructs the video that is not in the target area [15-17], and takes resolution reconstruction and spatial exchange matching of target data in the target area of basketball video. The calculation formula is as follows:

$$
S(\omega)=\frac{1}{K} \sum_{k=0}^{K-1} S_{k}(\omega) .
$$

In formula (8), $K$ represents the reconstruction coefficient and $S_{k}(\omega)$ represents the target data.

Assuming that the tracking targets in the target area of the basketball video are distributed linearly, the linear tracking target model in the public area of the basketball video is as follows:

$$
x(n)=\frac{1}{\sqrt{N}} A \sum_{k=0}^{N-1} X(k) \exp \frac{j 2 \pi k n}{N}, \quad n=0,1, \ldots, N-1 .
$$

Here, $j$ represents the number of image pixel node, $k$ represents the image pixel node, $A$ represents the amplitude of image reconstruction in basketball video, $N$ represents the sensor node in the collected basketball video, and $X(k)$ represents the target image pixel of the basketball video. Assuming that $A=\left\{a_{i, j}, 0<i, j<N\right\}$, the basketball video image can be decomposed into multimodule color map. Through the feature classification processing of the target data in the video, it can form the target pixel matrix $M \times N$ of basketball video.

Suppose the feature vector of the $i$-th pixel in the basketball video is $B=\left\{b_{j}(k)\right\}$; then when $0<j<N, 0<k<M$, and the initial pixel of the target data distribution in the basketball motion video is $\pi$, the probability distribution is $\pi=\left\{\pi_{i}, i=1,2, \ldots, n\right\}$ at this point. Using the grey normalization processing of grey neural network [18-20] and combining with the basketball video after template matching, it can obtain the single frame pixel feature set of the basketball video, which is as follows:

$$
s(k)=\left[s_{1}(k), s_{2}(k), \ldots, s_{m}(k)\right]^{T} .
$$

In the target area of basketball video, this algorithm matches the target image template of the block area and 
constructs the generation sequence of the target area in basketball video, which is as follows:

$$
\operatorname{Dif}\left(C_{1}, C_{2}\right)=N \min w\left(v_{i}, v_{j}\right) .
$$

In formula (11), $N=1,2, \ldots, T$ represents the feature collection sample of the target area in basketball video, $w\left(v_{i}, v_{j}\right)$ represents the pixel width of the basketball video, and the feature vector is composed of the eigenvalue of the target image in the target area of basketball video, thus achieving the acquisition of the target data of basketball video.

\subsection{Extraction of Key Feature Points of Basketball Video} Target. The following research is mainly to collect the target data of basketball video and extract the key feature points of the basketball video target [21]. Firstly, it is necessary to use the image degradation model to detect the feature of the target in the basketball video, combined with the improved grey neural network to optimally match the target image in the basketball video. If the threshold of the main feature sequence $h(j)$ of the target image in the basketball video is set as $h(j)$, the acquisition model of the target data in basketball video can be obtained. The formula is as follows:

$$
x(n)=\sum_{j=0}^{\infty} h(j) w(n-j) .
$$

Secondly, according to the motion curve of basketball video target image in three-dimensional space [22, 23], it can obtain the combined dispersion of target image in basketball video. The formula is as follows:

$$
\psi_{a, b}(t)=[U(a, b) \psi(t)]=\frac{1}{\sqrt{|a|}} \psi\left(\frac{t-b}{a}\right) .
$$

Here, $U(a, b)$ represents the similarity of pixels, and $\psi(t)$ represents the discrete features of the image.

The characteristic output matrix of basketball video target is as follows:

$$
X^{\prime}=\left[\begin{array}{cccc}
x_{11} & x_{12} & \cdots & x_{1 n} \\
x_{21} & x_{22} & \cdots & x_{2 n} \\
\vdots & \vdots & \cdots & \vdots \\
x_{(n-1) 1} & x_{(n-1) 2} & \cdots & x_{(n-1) n}
\end{array}\right] .
$$

Thirdly, after obtaining the feature vector of the basketball video, it can detect the moving image in the target area of the basketball video by template matching method [24] and get the fusion image, which is as follows:

$$
X_{t}=A X_{t-1}+t \text {. }
$$

In formula (15), $A$ represents the matching amplitude, $t$ represents the image frame, the coordinate point of the key feature point of the target in the basketball video is $X=\left[x_{t}, y_{t}\right]^{T}$. Assuming that the two most boundary target feature points in the target area of basketball video are $P E_{1}$ and $P E_{2}$, it can obtain the average matching pixel of the feature points.

$$
\begin{aligned}
& A=s\left[\begin{array}{cc}
\cos \theta & -\sin \theta \\
\sin \theta & \cos \theta
\end{array}\right], \\
& t=\left[\begin{array}{l}
t_{x} \\
t_{y}
\end{array}\right] .
\end{aligned}
$$

Finally, it also needs to match each feature point obtained above and geometrically describe the sparse items of the image based on the improved grey neural network and extract the motion feature of the target image in the basketball video by using the single frame visual difference analysis method.

$$
\frac{\partial u(x, y ; t)}{\partial t}=M \Delta_{s} u(x, y ; t)+N \Delta_{t} u(x, y ; d, t) .
$$

Here, $M$ and $N$, respectively, represent the common sparse item set of the image, and $\Delta_{t}$ represents the image area. Through the above operations, the key feature points of the basketball video target can be extracted.

2.4. Design of Target Tracking Algorithm of Basketball Video. Based on the improved grey neural network, it can reconstruct the target data of the target area in the basketball video, extract the key points of the motion video in the target area, and obtain the feature vectors of the basketball video. According to the improved grey neural network, the image feature points of the basketball video can be tracked and positioned; the tracking and positioning information can also be obtained, which is as follows:

$$
\theta(t)=-2 \pi K \ln \left(1-\frac{t}{a t_{0}}\right)+\theta_{0}
$$

Here, it can obtain $\theta_{0}=-2 \pi K \ln \left(1+T / 2 t_{0}\right)$ after the amplitude segmentation of image in the target area of the basketball video, in which $K$ represents the reconstruction coefficient. Then it is necessary to fuse the target image of basketball video through the fusion technology [25] and get the fused $n$ subimage blocks, so as to obtain the permutation matrix of the dynamic feature points of the basketball motion image. The permutation matrix is as follows:

$$
e_{m k}^{i j}= \begin{cases}-p_{k} \log \left(p_{k}\right), & p_{k} \neq 0, \\ 0, & p_{k}=0 .\end{cases}
$$

In formula (19), the probability value of dynamic characteristic distribution of basketball video is $p_{k}$, and then $m=1,2, \ldots, N$.

After extracting the implicit features of the target image in the target area of the basketball video and reconstructing the image superresolution of basketball video, it can obtain the output: 


$$
p_{i, j}(A)= \begin{cases}\frac{w_{i, j}}{w_{i}}, & \text { if } i \neq j \text { and } e_{i, j} \in A, \\ 0, & \text { if } i \neq j \text { and } e_{i, j} \notin A, \\ 1-\frac{\sum_{j: e_{i, \varepsilon}} w_{i, j}}{w_{i}}, & \text { if } i=j .\end{cases}
$$

Here, $w_{i, j}$ represents the weight of basketball video target image, $w_{i}$ represents the weight of pixel $i$, and $e_{i, j}$ represents the matching value of pixel.

The 3D feature reconstruction model of target area in basketball video is as follows:

$$
\operatorname{NLM}[g](i)=\sum_{j \in \Omega} w(i, j) g(j) .
$$

In formula (21), $w(i, j)$ represents the weight of image pixels $i$ and $j$, and $g(j)$ represents the sequence of pixel $j$. Assuming that the three-dimensional features of the target image of basketball video achieve uniform distribution [26], it can calculate the features of the target tracking image of basketball video.

$$
\mu_{p q}=\sum_{m=1}^{M} \sum_{n=1}^{N}(x-\dot{x})^{p}(y-\dot{y})^{q} f(x, y) .
$$

Here, $f(x, y)$ represents the scale information of target features, $(x-\dot{x})$ represents the feature quantity of tracking target $x$, and $(y-\dot{y})$ represents the feature quantity of tracking target $y$. According to the result of pixel reconstruction of basketball video, it can realize the reconstruction of dynamic feature in the target area of basketball video and achieve the design effect of target tracking algorithm of basketball video.

\section{Experimental Analysis}

In order to verify the effectiveness of the target tracking algorithm of basketball video based on the improved grey neural network in target tracking, this experiment tracks two video sequences with different video frame number and tracking area size. In the VS platform under Windows environment, the experiment uses $\mathrm{C}++$ language for programming, sets the experimental error threshold as 0.55 , and chooses three algorithms for comparison experiment, namely, the target tracking algorithm designed in this paper and the algorithm in [4] and in [5]. Three groups of basketball video sequences are selected for the test. And the image sequences of frames 23, 38, and 56 of the three groups of basketball video in a group of videos are collected as the sample sequences of the experimental test. This experiment mainly tests the basketball video target tracking effect of different algorithms under different basketball video frames, as shown in Figure 1.

According to Figure 1, when tracking the 23rd frame image of basketball video, the proposed algorithm, the algorithm in [4], and that in [5] can accurately track the basketball video target. When tracking the 38th frame image of basketball video, both the proposed algorithm and the algorithm in [4] can accurately track the basketball video target, while the algorithm in [5] has lost the basketball video target. When tracking the 56th frame image of the basketball video, both the proposed algorithm and the algorithm in [5] can accurately track the basketball video target, while the algorithm in [4] has errors in tracking the basketball motion video target. It can be seen that the proposed algorithm has a good effect on basketball video target tracking.

According to the image sequence of the basketball video, when tracking the image sequence of the 23rd, 38th, and 56th frames, this experiment tests the accuracy of the target tracking algorithm of basketball video based on the improved grey neural network. The results are shown in Figure 2.

As can be seen from the results in Figure 2, in the test of the target tracking algorithm of basketball video based on the improved grey neural network, when tracking the 23rd frame image of basketball video, the tracking accuracy is $80 \% \sim 85 \%$. When tracking the 38th frame of the basketball video, the tracking accuracy is between $84 \%$ and $91 \%$. When tracking the 56th frame of the basketball video, the tracking accuracy is between $85 \%$ and $98 \%$. Therefore, the target tracking algorithm designed in this paper has high accuracy in tracking the basketball video target. The target tracking algorithm designed in this paper can determine the actual position of the basketball video target by obtaining the target area of the basketball video, which can improve the tracking accuracy of the basketball video target.

In order to further verify the performance of the target tracking algorithm designed in this paper in the target tracking, this experiment collects three target images with different tracking areas of $600 * 400,480 * 320$, and $420 * 280$ and tests the target tracking effects of different algorithms in different tracking areas. The experimental results are shown in Figure 3.

According to Figure 3, it is known that when the tracking area of the target image is $600 * 400$, the proposed algorithm, the algorithm in [4], and that in [5] can accurately track the basketball video target. When the tracking area of the target image is $480 * 320$, the proposed algorithm can accurately track the basketball video target, while the algorithm in [4] has lost the basketball video target, and the algorithm in [5] has errors in tracking the basketball video target. When the tracking area of the target image is $420 * 280$, the proposed algorithm can still accurately track the basketball video target, while the algorithm in [4] and that in [5] have errors in tracking the basketball video target. Therefore, the target tracking effect of the proposed algorithm is good.

Under the condition of tracking target image sample sequences with different tracking area sizes, this experiment takes three target images with different tracking area sizes collected in basketball video as sample data and tests the tracking accuracy of the target tracking algorithm of basketball video based on improved grey neural network. The results are shown in Figure 4. 


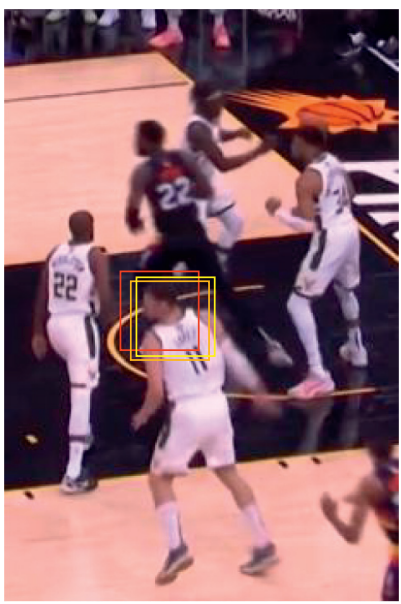

Frame 23 basketball video image sequence

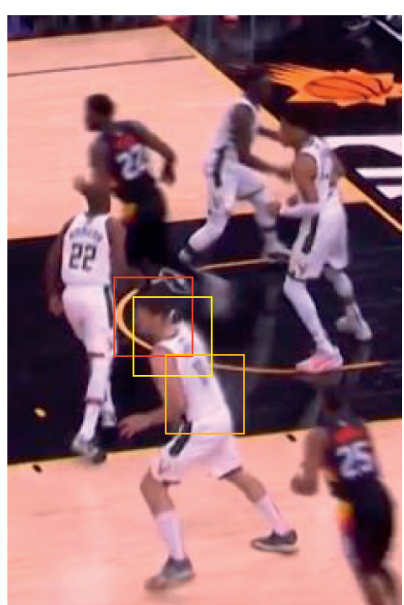

Frame 38 basketball video image sequence

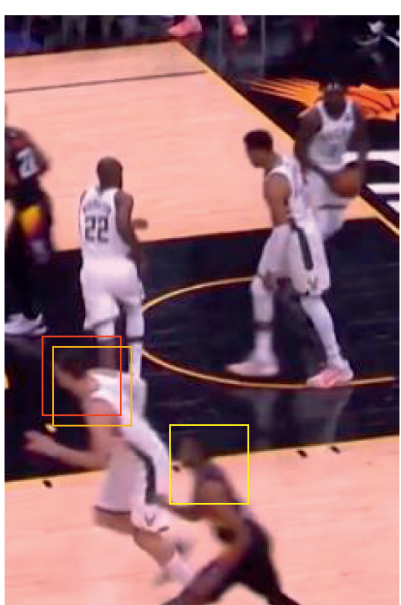

Frame 56 basketball video image sequence

_ The proposed algorithm

- Reference [4] algorithm

- Reference [5] algorithm

Figure 1: Target tracking effect of the algorithm under different basketball video frames.

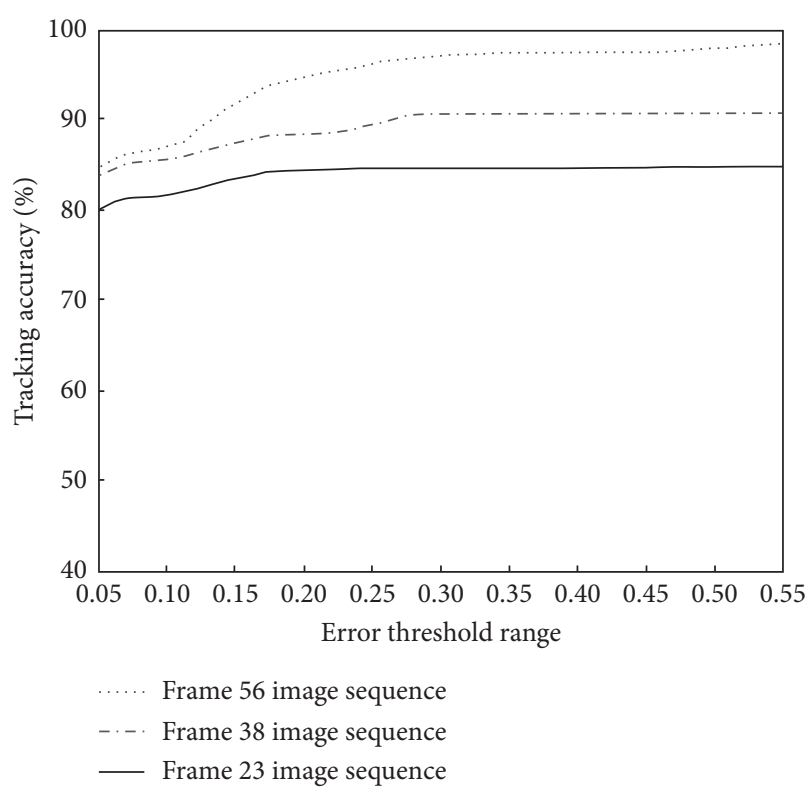

FIGURE 2: Test results of target tracking accuracy of basketball video with different frames.

As can be seen from the results in Figure 4, with the expansion of the error threshold range, the tracking accuracy of target image in different tracking areas gradually increases. When the tracking area of the target image is $600 * 400$, the target tracking accuracy of basketball video is between $86 \%$ and $98 \%$; when the tracking area of the target image is $480 * 320$, the target tracking accuracy of basketball video is between $83 \%$ and $95 \%$; when the tracking area of the target image is $420 * 280$, the target tracking accuracy of basketball video is between $80 \%$ and $90 \%$. Therefore, the target tracking algorithm designed in this paper has high accuracy in tracking the basketball video target. Among the three tracking areas with different sizes, this algorithm with the tracking area of $600 * 400$ has a higher target tracking accuracy of basketball video, which ensures the performance of the basketball video target tracking.

In order to further verify the anti-interference ability of the target tracking algorithm designed in this paper, the basketball video can be simulated by using $5.3 \mathrm{dmax} 3 \mathrm{D}$ simulation software in simulation environment, and the noise size of the image is set to $-0.003 \sim 0.003 \mathrm{~m}$, as shown in Figure 5.

In order to further verify the anti-interference ability of the target tracking algorithm designed in this paper, this experiment sets the noise with the size of $0.003 \sim 0.003 \mathrm{~m}$ in the simulation environment, compares the target tracking algorithm designed in this paper with the algorithm in [4] and that in [5], and obtains the target recognition rates of basketball video with different algorithms under noise interference, as shown in Table 1.

According to the data in Table 1, in the case of noise interference, when the amount of basketball video data is $500 \mathrm{MB}$, the average recognition rate of basketball video target of the algorithm in [4] is $74.8 \%$, and that of the algorithm in [5] is $65.2 \%$, while the average target recognition rate of basketball video target of the algorithm designed in this paper is as high as $90.6 \%$. Therefore, in the case of noise interference, the algorithm designed in this paper has a high target recognition rate and strong antiinterference ability. Because the algorithm designed in this paper processes the target area through grey level normalization, establishes the target area generation sequence, and collects the target data of the basketball video, it can effectively remove the image noise and redundant data, thus effectively improving the anti-interference ability of the algorithm designed in this paper. 


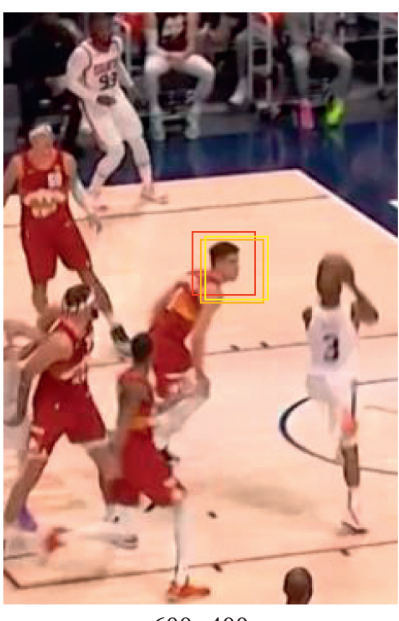

$600 * 400$

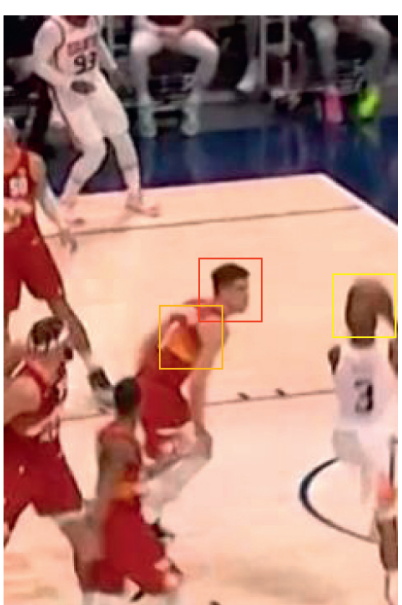

$480 * 320$

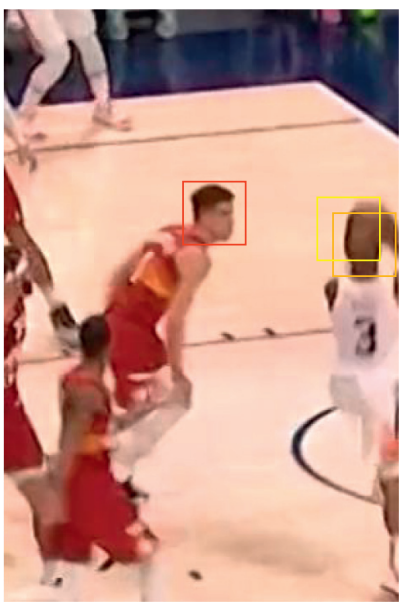

$420 * 280$

_ The proposed algorithm

Reference [4] algorithm

Reference [5] algorithm

FIgURE 3: Target tracking effect of the algorithm in different tracking areas.

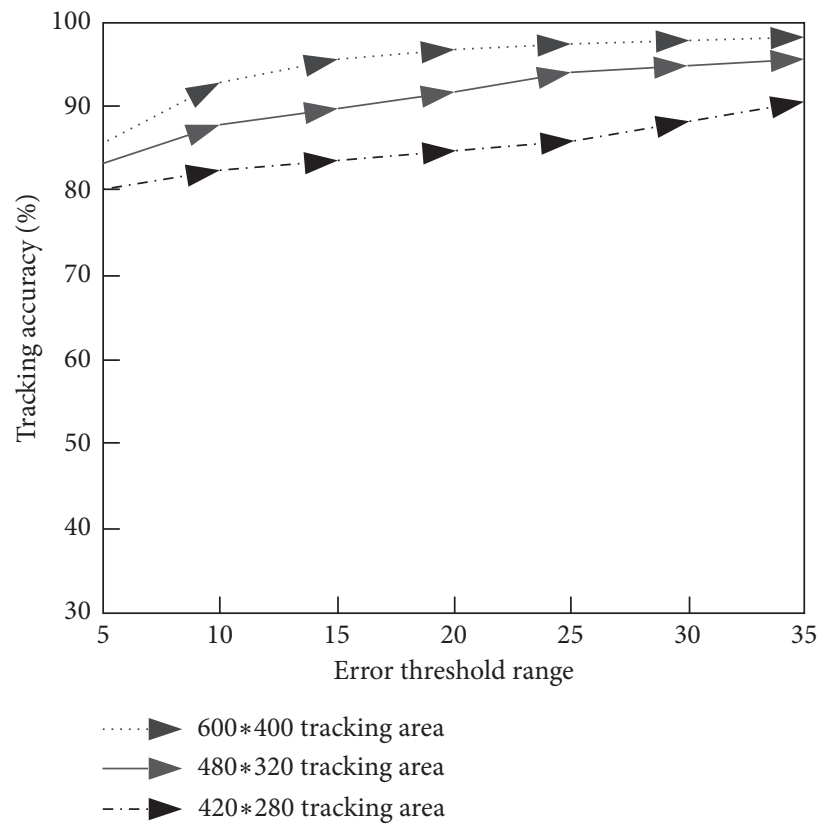

FIGURE 4: Test results of target tracking accuracy of basketball video in different areas.

On this basis, in order to verify the basketball video target tracking time of the target tracking algorithm designed in this paper, this experiment selects $500 \mathrm{MB}$ basketball video data and compares the basketball video target tracking time of the target tracking algorithm designed in this paper with that of the algorithm in [4] and that of the algorithm in [5]. The test results of basketball video target tracking time of different algorithms are shown in Table 2.

According to the data in Table 2, as the amount of basketball video data increases, the target tracking time of basketball video with different algorithms increases. When the amount of basketball video data is $500 \mathrm{MB}$, the basketball video target tracking time of the algorithm in [4] is $34 \mathrm{~s}$, and the basketball video target tracking time of the algorithm in [5] is $39 \mathrm{~s}$, while the basketball video target tracking time of the algorithm designed in this paper is only $22 \mathrm{~s}$. Therefore, the basketball video target tracking time of the algorithm designed in this paper is relatively short. The algorithm designed in this paper uses single frame visual difference analysis to extract the key feature points of the basketball video target and combines it with the improved grey neural 


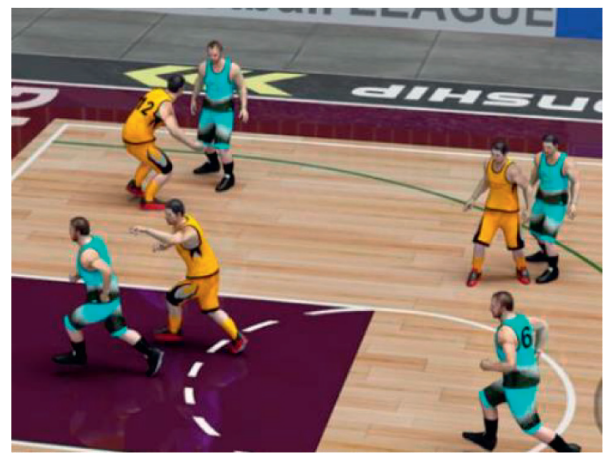

FIGURE 5: Simulation video of basketball.

TABLe 1: Target recognition rate of basketball video with different algorithms under noise interference.

\begin{tabular}{lccc}
\hline Data size of basketball video (MB) & The designed algorithm (\%) & The algorithm in [4] (\%) & The algorithm in [5] (\%) \\
\hline 100 & 89 & 78 & 69 \\
200 & 92 & 72 & 65 \\
300 & 95 & 75 & 63 \\
400 & 87 & 70 & 67 \\
500 & 90 & 62 & 67 \\
\hline
\end{tabular}

TABLE 2: Test results of target tracking time of basketball video with different methods.

\begin{tabular}{lccc}
\hline Data size of basketball video $(\mathrm{MB})$ & The designed algorithm $(\mathrm{s})$ & The algorithm in [4] $(\mathrm{s})$ & The algorithm in [5] $(\mathrm{s})$ \\
\hline 100 & 5 & 8 & 17 \\
200 & 9 & 23 & 20 \\
300 & 14 & 29 & 26 \\
400 & 19 & 34 & 31 \\
500 & 22 & 39 \\
\hline
\end{tabular}

network to track and locate the feature points of the basketball video target, thus effectively shortening the tracking time of the basketball video target.

This paper extracts the key feature points of basketball video target and tracks and locates the feature points of basketball video target combined with the improved grey neural network, thus effectively shortening the tracking time of basketball video target.

\section{Conclusion}

(1) For the target tracking of video images of basketball, different frames of video images are selected to complete the target tracking test. The results show that the proposed method can achieve high-precision target tracking of different images, and the tracking accuracy can be maintained between $80 \%$ and $98 \%$, which has application advantages compared with the literature algorithm.

(2) When the tracking region of the target image is different, the target tracking results of different algorithms are quite different. When the target image tracking area is large, the target tracking is not difficult, and both the literature algorithm and the proposed algorithm can complete the experimental task.
When the tracking area of the target image keeps shrinking, the recognition of target feature becomes more difficult, and the target tracking error of the literature algorithm appears obviously. But the proposed algorithm can still achieve the target tracking with high precision, and the accuracy can be maintained between $80 \%$ and $98 \%$.

(3) In the case of noise interference, the target recognition rate of basketball video designed in this paper is high.

When the amount of basketball video data is large, the target tracking time of basketball video designed in this paper is only $22 \mathrm{~s}$. It can be seen that the target tracking time of basketball video with the algorithm designed in this paper is short.

Based on improved grey neural network proposed in this paper, the target tracking algorithm of basketball video takes advantage of improved grey neural network. It reconstructs the basketball video target image by obtaining the basketball video target area, collecting the basketball video target data, and extracting the key feature points of the basketball video target, and it finally realizes the basketball video target tracking. Therefore, the target tracking algorithm designed in this paper has good tracking 
effect, which can effectively improve the tracking accuracy and ensure the performance of the algorithm.

\section{Data Availability}

The raw data supporting the conclusions of this article will be made available by the authors, without undue reservation.

\section{Conflicts of Interest}

The authors declare that they have no conflicts of interest regarding this work.

\section{Acknowledgments}

This study was supported by the Bidding Project of Jiaozuo Municipal Government Decision-Making Research in 2020- "Promoting the Study on the Spread of Taijiquan Culture along the One Belt and One Road" (JZZ2020061).

\section{References}

[1] Li Chen, "Research on target tracking algorithm of fast human moving in video image," Modern Electronic Technique, vol. 42, no. 3, pp. 49-51, 2019.

[2] J. Mao, "A video target tracking algorithm based on wireless sensor networks," Control Engineering, vol. 25, no. 1, pp. 71-75, 2018.

[3] Y. Li, J. Lu, H. Li, W. Zhang, and Y. Zhang, "Research on target tracking algorithm from fisheye camera based on compressive sensing," Journal of Electronics and Information Technology, vol. 40, no. 5, pp. 1242-1249, 2018.

[4] B. Yang and T. Wang, "Implementation of target tracking algorithm of embedded basketball video based on DSPFPGA," Natural Science Journal of Xiangtan University, vol. 40, no. 6, pp. 104-108, 2018.

[5] J. Wang and X. Hou, "Design of target tracking algorithm of sports video based on optimized particle filter," Machine Tool \& Hydraulics, vol. 46, no. 6, pp. 164-169, 2018.

[6] D. Wang, H. Fang, Y. Liu, S. Wu, Y. Xie, and H. Song, "A target tracking algorithm of panoramic video based on improved RT-MDNet," Journal of Harbin Institute of Technology, vol. 52, no. 10, pp. 152-160+174, 2020.

[7] S. Javed, A. Mahmood, J. Dias, and N. Werghi, "Robust structural low-rank tracking," IEEE Transactions on Image Processing, vol. 29, pp. 4390-4405, 2020.

[8] C. Fang, J. Huang, and K. Cuan, "Comparative study on poultry target tracking algorithms based on a deep regression network," Biosystems Engineering, vol. 190, pp. 176-183, 2020.

[9] P. Zheng, H. Bai, Z. Li, and G. Hongwei, "Design of accurate detection and tracking algorithm of moving target under jitter interference," Chinese Journal of Scientific Instrument, vol. 40, no. 11, pp. 90-98, 2019.

[10] T. Wang, "Unsupervised video multi-target tracking based on fast resampling particle filter," The Journal of Supercomputing, vol. 76, no. 2, pp. 1293-1304, 2020.

[11] Z Cai and H Feng, "Target tracking based on improved square root cubature particle filter via underwater wireless sensor networks," IET Communications, vol. 13, 2019.

[12] Yi Liu, "Research on improved particle filter model technology for target tracking of moving video," Modern Electronics Technique, vol. 42, no. 3, pp. 65-67+72, 2019.
[13] A. Silva Ramos, C. Hora Fontes, A. Magdiel Ferreira et al., "Somatic cell count in buffalo milk using fuzzy clustering and image processing techniques," Journal of Dairy Research, vol. 88, no. 1, pp. 69-72, 2021.

[14] Y. Kim and G. Dodbiba, "A novel method for simultaneous evaluation of particle geometry by using image processing analysis," Powder Technology, vol. 393, pp. 60-73, 2021.

[15] Y. Qi and M. Wang, "Tracking algorithm of moving target based on LBP and kernel correlation filter," Infrared Technology, vol. 41, no. 6, pp. 572-576, 2019.

[16] S. Unar, X. Wang, C. Wang, and Y. Wang, "A decisive content based image retrieval approach for feature fusion in visual and textual images," Knowledge-Based Systems, vol. 179, no. SEP.1, pp. 8-20, 2019.

[17] F. Yuan, G. Li, X. Xia, B. Lei, and J. Shi, "Fusing texture, edge and line features for smoke recognition," IET Image Processing, vol. 13, no. 14, pp. 2805-2812, 2019.

[18] W. Ou, H. Yin, H. Xu, and Z. Liu, "A multi-odject tracking algorithm based on trajectory reconstruction on multi-egocentric video," CAAI Transactions on Intelligent Systems, vol. 14, no. 2, pp. 246-253, 2019.

[19] K. Z. Rajab, B. Wu, P. Alizadeh, and A. Alomainy, "Multitarget tracking and activity classification with millimeterwave radar," Applied Physics Letters, vol. 119, no. 3, Article ID 034101, 2021.

[20] D. Yao, C. Dou, D. Yue, Z Nan, and Z Tingjun, “Adaptive neural network consensus tracking control for uncertain multi-agent systems with predefined accuracy," Nonlinear Dynamics, vol. 101, no. 1, pp. 2249-2262, 2020.

[21] X. Yu and S. Li, "Research on tracking algorithm of moving target based on multi-step prediction and Mean-Shift," Infrared Technology, vol. 40, no. 12, pp. 1182-1187, 2018.

[22] J. Li, Z. Shang, and H. Liu, "Target tracking algorithm of correlation filtering moving based on multi-layer convolution feature," Computer Science, vol. 046, no. 7, pp. 252-257, 2019.

[23] H. Zhang, P. Rao, H. Xia, D. Weng, X. Chen, and Y. Li, "Modeling and analysis of infrared radiation dynamic characteristics for space micromotion target recognition," Infrared Physics \& Technology, vol. 116, no. 3, Article ID 103795, 2021.

[24] C. Chao, "Target tracking algorithm involving the Gaussian mixture model and weighted likelihood computer engineering and applications," Computer Engineering and Applications, vol. 55, no. 12, pp. 124-131, 2019.

[25] P. Jia and W. Jia, "Research on coal mine multi-target trajectory tracking algorithm," Computer Engineering and Applications, vol. 54, no. 2, pp. 227-232, 2018.

[26] B. Wei, P. Ge, H. Wu, G. Wang, and W. Han, "Object tracking algorithm based on static-adaptive appearance model correction," Computer Applications, vol. 038, no. 4, pp. 1170-1175, 2018. 